\title{
Emulating extreme velocities of mobile LTE receivers in the downlink
}

\author{
José Rodríguez-Piñeiro ${ }^{*}$, Martin Lerch², José A. García-Naya', Sebastian Caban², Markus Rupp² \\ and Luis Castedo ${ }^{1}$
}

\begin{abstract}
Long-Term Evolution (LTE) is expected to substitute the Global System for Mobile (GSM) Communications as the radio access technology for railway communications. Recently, considerable attention has been devoted to high-speed trains since this particular environment poses challenging problems in terms of performance simulation and measurement. In order to considerably decrease the cost and complexity of high-speed measurement campaigns, we have proposed a technique to induce effects caused by highly time-varying channels on Orthogonal FrequencyDivision Multiplexing (OFDM) signals while conducting measurements at low speeds. In this work, we illustrate the performance of this technique by comparing the results of LTE measurements at different velocities in a controlled measurement environment. Additionally, we validate this technique by means of simulations, considering one of the scenarios defined as part of the Winner Phase II Channel Models, specifically designed for high-speed train scenarios.
\end{abstract}

Keywords: LTE; OFDM; Measurement; High speed

\section{Introduction}

Over the last few years, broadband communication between nodes moving at high speeds has attracted special attention. One of the most relevant research topics in this field is the modeling of the High-Speed Train (HST) channel. Nowadays, the most widely used communication system between trains and the elements involved in operation, control, and intercommunication within the railway infrastructure is based on the Global System for Mobile (GSM) Communications. This technology, namely, the GSM for Railways (GSM-R), is not well-suited for supporting advanced services such as automatic pilot applications or provisioning broadband services to the train staff and passengers. Besides trains, the increasing number of broadband services available for mobile devices motivated the migration from third-generation mobile networks to fourth-generation ones, mainly Long-Term Evolution (LTE). Therefore, LTE seems to be a good candidate to substitute the GSM as the fundamental technology for railway communications.

\footnotetext{
${ }^{*}$ Correspondence: j.rpineiro@udc.es

1 Department of Electronics and Systems, University of A Coruña, Facultade de Informática, Campus de Elviña, A Coruña 15071, Spain

Full list of author information is available at the end of the article
}

Several radio channel models have been proposed for moving radio interfaces, such as the International Telecommunication Union (ITU) channel models [1]. The first channel modeling approach for high-speed train scenarios was proposed by Siemens in 2005 [2]. More recent proposals are the Winner Phase II Model (high-speed moving networks were included in [3]) and the radio channel models approved by the ITU Radiocommunication (ITU-R) Sector for the evaluation of IMT-Advanced Technologies (high-speed train scenarios were explicitly considered in [4]). However, only a few results based on empirically obtained data which can validate the aforementioned channel models are available. One example of high-speed train channel modeling contributions based on the results obtained by means of measurement campaigns is the propagation path-loss model proposed in [5]. The same experimental results were also used for the definition of the large-scale model proposed in [6]. An efficient channel sounding method for high-speed train scenarios using cellular communications systems is proposed in [7] by considering Wideband Code Division Multiple Access (WCDMA) signals.

One of the reasons that explains the low number of measurement campaigns in high-speed environments is their complexity and cost. Furthermore, it is not possible,

\section{Springer}


in most cases, to measure at high speeds in controlled environments in a reproducible and repeatable way. In addition, measuring in high-speed trains demands for specific hardware and software solutions (see [8]). In order to address those problems, we proposed a technique to induce the effects caused by highly time-varying channels in Orthogonal Frequency-Division Multiplexing (OFDM) signals while conducting the measurements at much lower speeds [9]. This technique consists basically in reducing the subcarrier spacing of the OFDM signal by scaling down the bandwidth of the whole OFDM signal. More specifically, we propose to interpolate the transmit OFDM signal in the time domain before its transmission. The time-interpolated signal still conveys exactly the same information as the original one but with a reduced subcarrier spacing, thus artificially increasing the sensitivity to the Inter-Carrier Interference (ICI). For example, if we time interpolate the transmit OFDM signal by a factor $I$, the subcarrier spacing will be reduced by the same factor $I$, which is similar to what would happen if the transmission was conducted at $I$ times the original speed.

In [9], we have proposed this novel methodology for evaluating the performance of OFDM transmissions in real-world scenarios affected by large Doppler spreads while conducting the measurements at low speeds. More specifically, in [9], we considered the transmission of standard-compliant WiMAX Mobile (IEEE 802.16e) signals, both through simulations and realistic outdoor measurements. When conducting measurements with movement in realistic environments and using off-theshelf vehicles (e.g., cars, trains), the repeatability of the results is basically lost because the following issues arise:

- It is extremely difficult to keep the vehicle speed constant during the measurements.

- Reaching high-speed values with experimental user equipment is expensive and sometimes even not possible in realistic environments.

- Impacts from measurement control can always arise (e.g., another vehicle driving nearby) leading to non-repeatable results.

- Modifications in the measurement scenarios (e.g., the path of the road is modified, a huge vehicle is parked close to the measurement path) hinder fair comparisons with future measurements.

- It is quite challenging to drive several times along exactly the same path.

In order to validate the technique proposed in [9] while, at the same time, overcoming all the abovementioned issues found when conducting non-repeatable measurements using off-the-shelf vehicles, we performed the assessment employing a setup that allows for repeatable measurements at different speeds up to $200 \mathrm{~km} / \mathrm{h}$ and considered LTE as the wireless communication standard. This setup consists in an antenna that is rotated around a central pivot at a constant speed, allowing for validating our previously proposed technique under repeatable as well as controlled conditions.

We complete the validation by means of simulations considering a channel model suited for high-speed train scenarios. More specifically, we consider the channel model associated with the D2a link of the D2 scenario of the Winner Phase II Channel Models [3]. Notice that our intention is not to model the measurement environment using a channel model existing in the literature. Our intention is to prove the validity of our results in a perfectly repeatable (and controlled) environment which is widely accepted for simulating high-speed train conditions. Therefore, measurement and simulation scenarios correspond to very different wireless communication environments since the simulations consider a rural macro-cell with Line-of-Sight (LoS) between transmitter and receiver, while an outdoor-to-indoor communication without LoS is considered for the measurements.

The presented results, which were obtained under repeatable conditions, both, through measurements and simulations, show that our technique induces highly timevarying channels with excellent agreement.

The rest of the paper is organized as follows. Section 2 explains the proposed time interpolation technique to induce high-speed effects on OFDM signals while conducting measurements at much lower speeds. Section 3 describes the setup and measurement methodology for measurements as well as for simulations. Different figures of merit for the results are also introduced. Section 4 presents both, measurement and simulation results, and shows the excellent agreement obtained with the proposed technique. Finally, Section 5 is devoted to conclusions.

\section{Emulating high speeds by time interpolation}

Let us consider an OFDM modulation where $N$ subcarriers are multiplexed to construct each OFDM symbol. OFDM symbols are cyclically extended by adding $N_{g}$ samples, thus having a total length of $N_{t}=N+N_{g}$ samples. Accordingly, the $k$-th OFDM symbol can be represented as:

$$
\mathbf{s}_{k}=\mathbf{G}_{1} \mathbf{F}^{H} \mathbf{x}_{k}
$$

where $\mathbf{x}_{k}$ is a $N \times 1$ vector containing the constellation symbols corresponding to the transmitted subcarriers in the $k$-th symbol, $\mathbf{F}$ is the standard $N \times N$ DFT matrix, $\mathbf{G}_{1}$ is a $N_{t} \times N$ matrix which cyclically extends the OFDM symbol, and $\mathbf{s}_{k}$ is a $N_{t} \times 1$ vector with the transmitted OFDM symbol in the time domain. This signal is transmitted at a sampling period $T_{s}$, hence creating symbols with a duration $T_{t}=T_{s} N_{t}$ and a bandwidth $F_{s}=1 / T_{s}$. When 
transmitting $\mathbf{s}_{k}$ over a time-varying channel and assuming a sufficiently long cyclic prefix, the received signal is [10]:

$$
\mathbf{r}_{k}=\mathbf{F G}_{2}\left(\mathbf{H}_{k}^{(t)} \mathbf{G}_{1} \mathbf{F}^{H} \mathbf{x}_{k}+\mathbf{n}_{k}\right)=\mathbf{H}_{k} \mathbf{x}_{k}+\mathbf{w}_{k},
$$

where $\mathbf{H}_{k}^{(t)}$ is a $N_{t} \times N_{t}$ matrix with the time-varying channel impulse response experienced by $\mathbf{s}_{k}, \mathbf{G}_{2}$ is a $N \times N_{t}$ matrix to remove the cyclic prefix, $\mathbf{n}_{k}$ is a $N_{t} \times 1$ vector with uncorrelated complex-valued white Gaussian noise entries with power $\sigma^{2}, \mathbf{H}_{k}$ is a $N \times N$ matrix with the channel frequency response, and $\mathbf{w}_{k}$ is a $N \times 1$ vector containing uncorrelated complex-valued white Gaussian noise entries with variance $\sigma^{2}$.

If the channel is time invariant, $\mathbf{H}_{k}$ will be a diagonal matrix. In time-selective channels, however, non-zero entries will appear outside the main diagonal of $\mathbf{H}_{k}$ and ICI arises in the received signal. The amount of ICI relates to the normalized Doppler spread of the channel, which is given by $D_{n}=f_{d} T, f_{d}$ being the maximum Doppler frequency and $T=T_{s} N$ the duration of the OFDM symbol excluding the cyclic prefix. As proposed in our previous work [9], parameter $T$ can be adjusted by time interpolation by a factor $I$, yielding an OFDM symbol duration $T^{I}=I T_{s} N$. Therefore, given the actual velocity $v$ of the mobile receiver, the normalized Doppler spread, impacting the time-interpolated OFDM signal can be written as:

$$
D_{n}^{I}=f_{d} T^{I}=f_{d} I T_{s} N=\frac{T_{s} N I f_{c} v}{c}=\frac{T_{s} N f_{c}}{c} v^{I},
$$

with $f_{c}$ the carrier frequency, $c$ the speed of light, and $v^{I}=$ I $v$ the emulated speed as a result of an actual measurement speed $v$ and an interpolation factor $I$. Consequently, enlarging the symbol length $T^{I}$ by adjusting $I$ allows for the emulation of a velocity $v^{I}$ while conducting measurements at an actual speed $v$. Notice that this procedure is also valid for time decimation of the signal, simply taking $0<I<1$, leading to an emulated speed lower than the actual one. Finally, note also that $I$ does not have to be an integer value. Fractional time interpolation and decimation factors are also possible, hence providing a great flexibility for adjusting $v^{I}$ from $v$.

In our setup, time interpolation factors $I \geq 1$ were applied to standard-compliant downlink LTE OFDM signals before the over-the-air transmission to emulate situations with $I$ times higher velocity than the actual receiver speed. The same principle was considered for the simulations.

\section{Evaluation setup and procedure}

We use the measurement setup shown in Figure 1 (measurement branch) to test the proposed technique of emulating high speeds by time interpolation of OFDM signals. The setup consists of:

1. Signal generation (transmitter side) and signal processing (receiver side): at the transmitter side, standard-compliant LTE subframes are generated using the LTE Downlink Link-Level Simulator developed at the TU Wien [11]. At the receiver side, the same simulator is used to process the received signals and to estimate the following figures of merit: uncoded Bit Error Ratio (BER), coded BER, Error Vector Magnitude (EVM), and Signal-to-Noise Ratio (SNR).

2. Time interpolation and time decimation: the signal is time-interpolated by a factor $I$ at the transmitter and decimated by the same factor $I$ at the receiver side. This way, we emulate a Doppler spread similar to that obtained with a speed increase by a factor of $I$.

3. Signal transmission and signal reception: signals are transmitted over the air by using a testbed developed

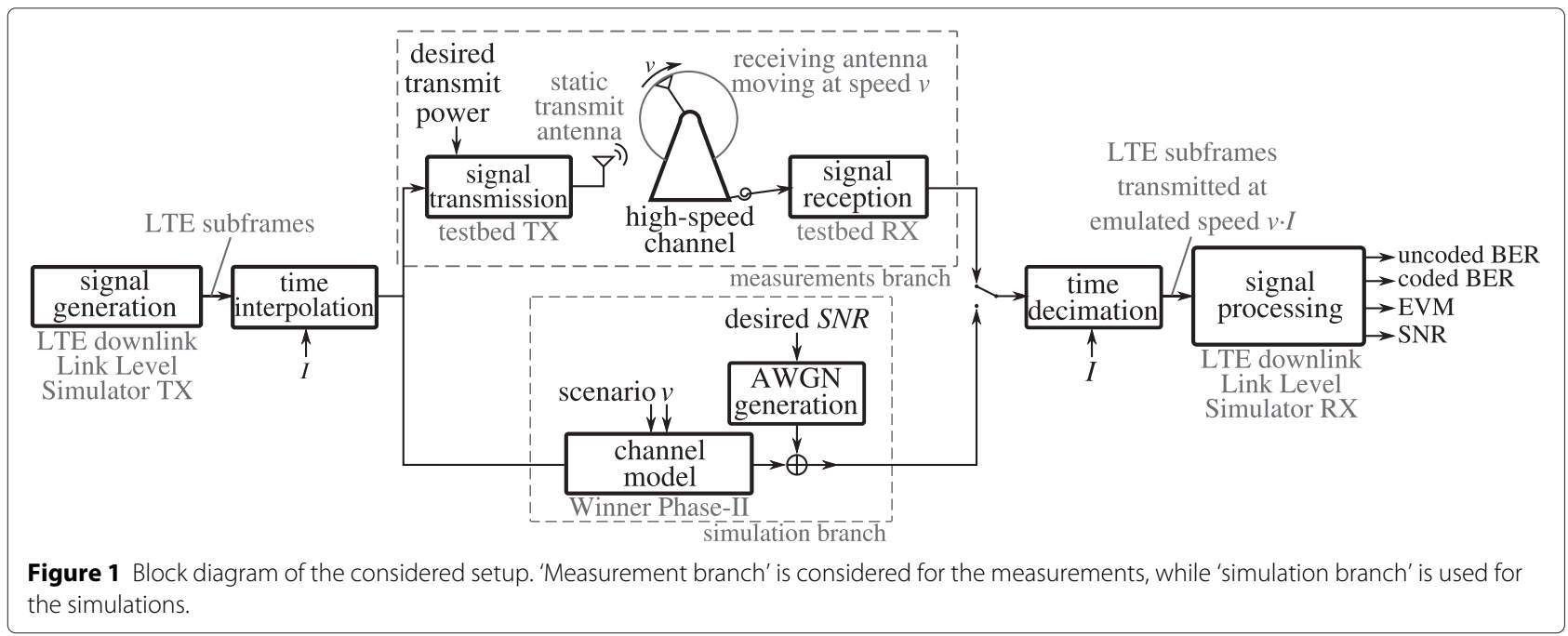


at the TU Wien [12,13]. The testbed transmitter is placed outdoors on a roof of a building in downtown Vienna, Austria. The receiver is placed indoors in the fifth floor of an adjacent building at a distance of 150 $\mathrm{m}$ (see Figure 2), hence recreating an infrastructure-to-vehicle scenario. Note that no feedback channel was used in our experiments. Consequently, no adaptive modulation and coding schemes were applied. Channel Quality Indication (CQI) values were fixed in advance. Notice also that the testbed is equipped with a highly precise time and frequency synchronization system based on Global Positioning System (GPS)-disciplined rubidium oscillators and a custom-made synchronization unit [14]. As a result, we can assume perfectly time and frequency synchronized measurements. Thus, the results are not affected by time or frequency offsets due to imperfect synchronization.

4. Generation of high-speed conditions: high-speed conditions are generated by transmitting from a static transmit antenna to a receive antenna that is rotated around a central pivot in a controlled and repeatable way $[15,16]$ (see Figure 3). Different channel realizations are created by measuring at different initial positions of the receive antenna. Note that the trajectory of the antenna is well approximated by a straight line since the diameter of the trajectory is 2 $\mathrm{m}$ and each LTE subframe is $1 \mathrm{~ms}$ long (see Figure 4).

\subsection{Measurement setup}

In order to evaluate the impact of high-speed conditions on LTE transmissions, actual velocities ranging from 50 to $200 \mathrm{~km} / \mathrm{h}$ were considered. Furthermore, interpolation factors of $I=1$ (no interpolation), $I=2$, and $I=3$ were used for generating Doppler spreads equivalent to those associated to velocities ranging from 50 to $600 \mathrm{~km} / \mathrm{h}$. Note that it is possible to generate exactly the same Doppler spread value from different combinations of the actual measurement velocity and the interpolation factor (see Table 1). We considered this fact to show that our technique allows for the evaluation of wireless communication systems at high speeds while measuring at much lower speeds. In order to do that, we generated the same Doppler spread by means of different velocities and interpolation factors, and then, we compared the obtained results. Table 1 shows the combinations of actual speeds and interpolation factors which lead to equal Doppler spreads (each row of the table corresponds to a different Doppler spread factor).

\subsection{Measurement procedure}

Several measurements are conducted for each velocity and interpolation factor. More specifically, three different positions of the whole receiver (including the antenna rotation unit) along its rails (see Figure 3 ) are considered. For each of these positions, 10 measurements per velocity and interpolation factor are carried out, each starting at a given angular shift on the circumference defined by the receive antenna rotating around a central pivot. Therefore, 30 different channel realizations are measured in total for each pair of velocity and interpolation factor. Furthermore, the whole set of measurements is repeated for each SNR value, among those specified in Table 2. The transmit power employed at the testbed transmitter

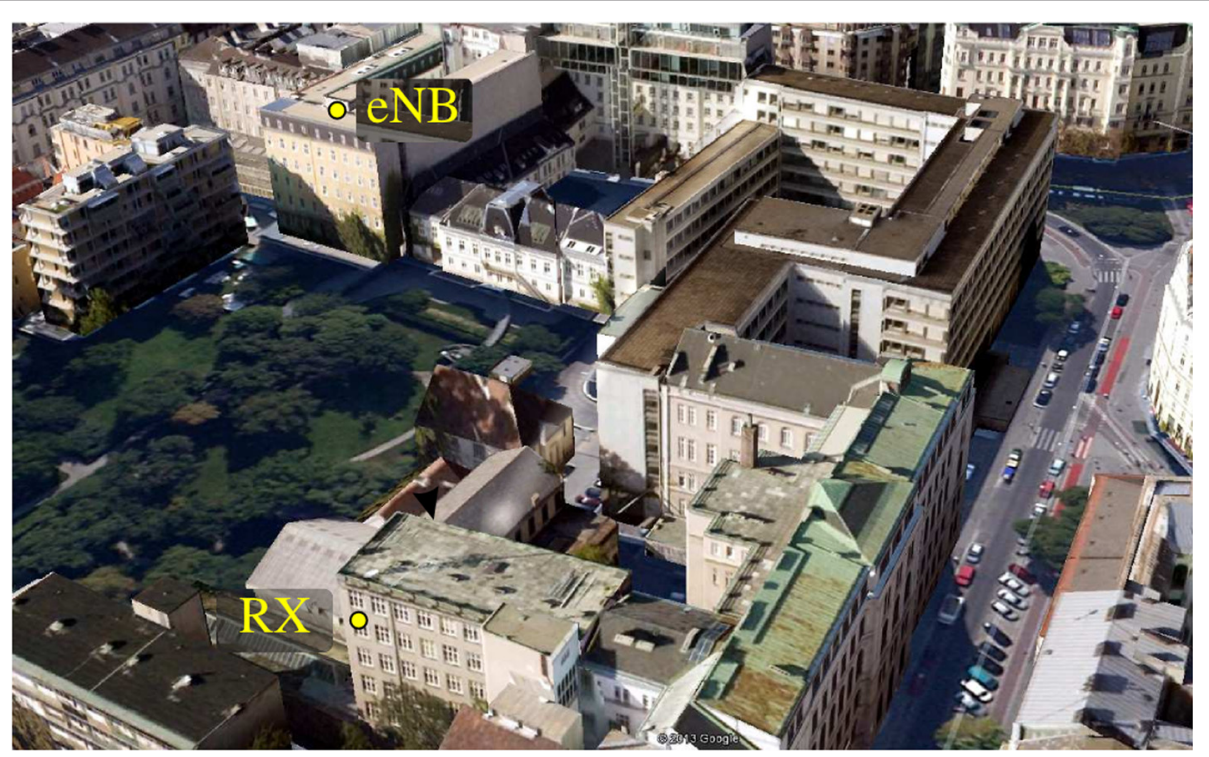

Figure 2 Location of the transmitter and the receiver. Note that the transmitter is installed outdoors on a roof of a building, while the receiver is placed indoors in the fifth floor of an adjacent building. The distance between transmitter and receiver is approximately $150 \mathrm{~m}$. 


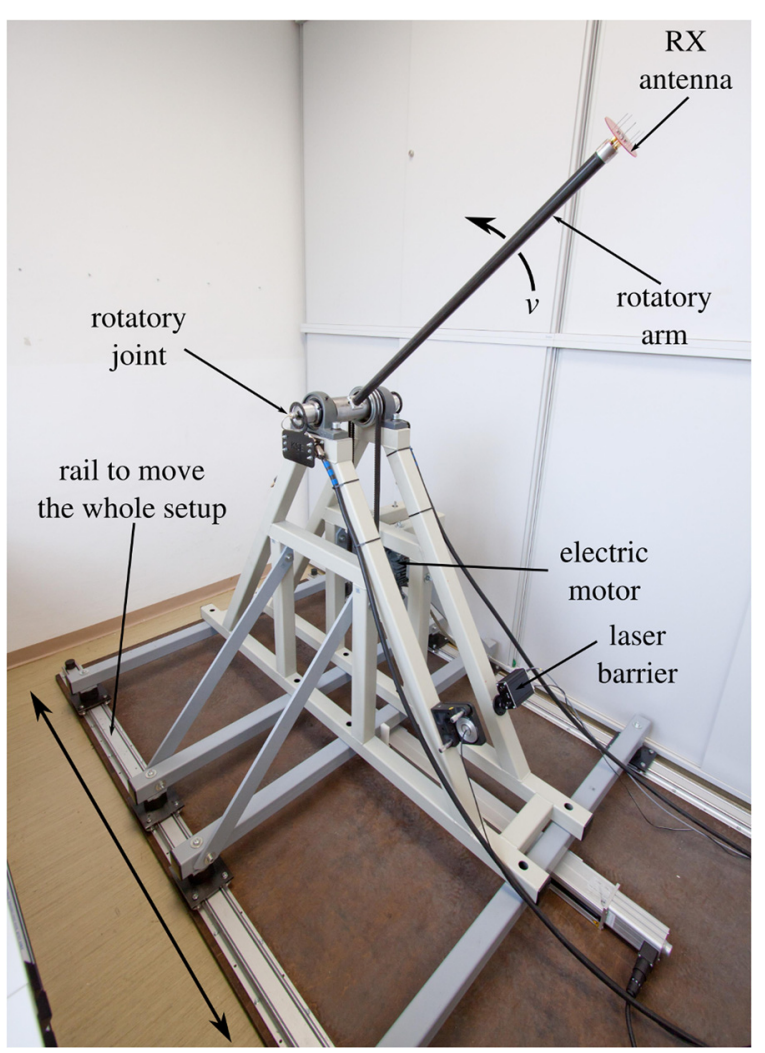

Figure 3 Setup used for generating high-speed conditions. The receive antenna is rotated around a central pivot, generating high-speed conditions in a controlled and repeatable way.

is modified in order to achieve different average SNR values.

In order to be able to compare the results gathered from different interpolation factors, we have to ensure that the receive antenna trajectory, spectrum usage, and average OFDM symbol energy remain constant for each emulated velocity $v \cdot I$ :

1. Equal receive antenna trajectory: in order to maintain a constant Doppler spread, the rotation speed has to be divided by the interpolation factor (see Table 1). Therefore, as it is shown in Figure 4, the trajectory of the receive antenna during the transmission of one LTE subframe does not vary when changing the interpolation factor and antenna velocity as long as the emulated speed is the same.

2. Equal spectrum usage: when a subframe is interpolated by a factor of $I$, its bandwidth is decreased by the same factor, which in principle reduces the effect of the channel frequency diversity. In order to experience the same spectrum, I replicas of the interpolated signal are transmitted to ensure that the whole frequency range of the original signal is used. The results are then averaged. Figure 5 shows an example of this procedure for $I=2, I=3$, and for an arbitrary interpolation factor.

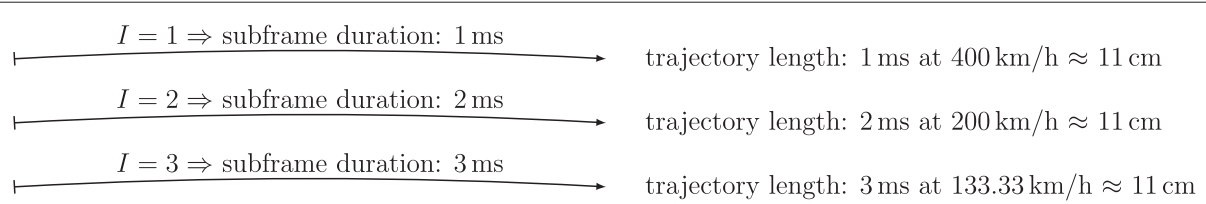

Figure 4 Trajectory followed by the antenna during the acquisition of a single LTE subframe. An emulated speed of $400 \mathrm{~km} / \mathrm{h}$ was used and the three interpolation factors $/=\{1,2,3\}$ were considered. Evaluating the results at a speed value of $400 \mathrm{~km} / \mathrm{h}$ can be done 1 ) by measuring at the actual velocity of $400 \mathrm{~km} / \mathrm{h}$ without time interpolation $(I=1) ; 2)$ by measuring at half the speed with $/=2$; and 3 ) by measuring at $400 / 3=133.33$ $\mathrm{km} / \mathrm{h}$ with $/=3$. In all cases, the length of the trajectory described by the antenna is the same (close to $11 \mathrm{~cm}$ ) and can be approximated by a straight line since the antenna describes a circle with a diameter of $2 \mathrm{~m}$ while rotating around the central pivot. 
Table 1 Emulated speed values (expressed in $\mathrm{km} / \mathrm{h}$ ) that can be obtained from more than an actual velocity $v$

\begin{tabular}{llll}
\hline Emulated speed, $\boldsymbol{v}^{\boldsymbol{I}}=\boldsymbol{I} \boldsymbol{v}$ & $\boldsymbol{I}=\mathbf{1}$ & $\boldsymbol{I}=\mathbf{2}$ & $\boldsymbol{I}=\mathbf{3}$ \\
\hline 100 & $v=100$ & $v=50$ & - \\
150 & $v=150$ & $v=75$ & $v=50$ \\
200 & $v=200$ & $v=100$ & $v=66.6$ \\
250 & - & $v=125$ & $v=83.3$ \\
300 & - & $v=150$ & $v=100$
\end{tabular}

Notice that these are the speed values considered in the error curves in Figures $6,7,8,9,10$, and 11 .

3. Equal average transmit energy per OFDM symbol: in order to preserve the average energy per OFDM symbol, the interpolated signals are scaled in amplitude by a factor of $\sqrt{I}$ before being transmitted.

Notice that our main objective is to validate the proposed technique considering a realistic scenario while, at the same time, keeping its complexity under reasonable terms, hence simplifying the validation process and facilitating the comprehension of the results. Therefore, we consider over-the-air standard-compliant LTE transmissions as a good example of the state of the art in wireless communications. However, due to the abovementioned simplicity reasons, we restrict the evaluation to a singleuser scenario without feedback channel, so modulation and coding scheme values are fixed beforehand instead of being adapted according to the channel quality.

Table 2 Main parameters for measurements as well as for simulations

\begin{tabular}{|c|c|c|c|}
\hline Parameters & \multicolumn{3}{|l|}{ Values } \\
\hline Bandwidth, $F_{s}(\mathrm{MHz})$ & \multicolumn{3}{|c|}{15.36 (9 occupied) } \\
\hline FFT size, $N$ & \multicolumn{3}{|l|}{1,024} \\
\hline Used subcarriers & \multicolumn{3}{|c|}{600 (excluding DC) } \\
\hline Velocities, $v(\mathrm{~km} / \mathrm{h})$ & \multicolumn{3}{|c|}{$50,66.6,75,83.3,100,125,150$, and 200} \\
\hline Carrier frequency, $f_{C}(\mathrm{GHz})$ & \multicolumn{3}{|l|}{2.5} \\
\hline Interpolation factors, I & \multicolumn{3}{|c|}{1,2 , and 3} \\
\hline $\mathrm{SNR}(\mathrm{dB})$ & \multicolumn{3}{|c|}{$38,31,21,11,1$, and $\infty$ (simulations only) } \\
\hline CQI values & 1 & 8 & 12 \\
\hline Modulation & 4-QAM & 16-QAM & 64-QAM \\
\hline Code rate & 0.076 & 0.48 & 0.65 \\
\hline Efficiency & 0.1523 & 1.9141 & 3.9023 \\
\hline Source bits & 1,192 & 15,288 & 31,192 \\
\hline Coded bits & 16,000 & 32,000 & 48,000 \\
\hline Peak throughput (Mbit/s) & 1.192 & 15.288 & 31.192 \\
\hline
\end{tabular}

Although three CQI values were evaluated and considered throughout the text to validate the proposed technique, results in the figures only consider $\mathrm{CQI}=12$.
Each LTE frame in the Frequency-Division Duplex (FDD) mode consists of ten subframes, each one following a different structure depending on the subframe index. For example, synchronization signals are always allocated in the first and sixth subframes, while broadcast channels are included, if necessary, in the first subframe. In order to make the comparison between channel realizations independent of the subframe structure, we always transmit the seventh subframe, considering only pilot and data symbols in subsequent evaluations. Consequently, we ensure the same payload for each channel realization, which enables us to fairly compare the results of different measurements as the subframe structure is kept invariant all the time. More specifically, the number of data symbols that can be transported in the seventh subframe according to the system configuration is 8,000 , which leads to the number of coded bits, source bits, and peak throughput values specified in Table 2.

\subsection{Simulation environment}

We employ the setup shown in Figure 1 (simulation branch) to validate through simulations our technique of emulating high speeds by time interpolation. We select noise variance values that lead to the same SNR values estimated from the measurements. A channel model suited for high-speed train scenarios was considered, namely, the model defined by the D2a link of the D2 scenario of the Winner Phase II Channel Models [3]. This scenario models the channel between an antenna placed at the roof of a train carriage and a network of fixed eNodeBs installed in a rural environment, which usually leads to LoS propagation conditions. Speeds up to $350 \mathrm{~km} / \mathrm{h}$ can be considered for the train movement.

Notice that the considered channel model does not intend to reproduce the wireless channel observed in the measurements. Our intention is to complement the evaluation of the proposed technique in a simulation environment while, at the same time, avoiding the process of characterizing the doubly selective wireless channel observed in the measurements, which is not the intention of this work. Finally, we selected the aforementioned channel model because it is specifically designed to model an outdoor-to-outdoor high-speed scenario with LoS between transmitter and receiver, while the measurement environment corresponds to an outdoor-to-indoor scenario with Non-Line-of-Sight (NLoS) between them.

\subsection{Simulation procedure}

The number of evaluated subframes by simulation was the same considered when measuring, i.e., 30 channel realizations for each velocity and interpolation factor pair. The values of SNR, velocity, and interpolation factors were also kept unchanged. We also guarantee an equal spectrum usage as well as equal average transmit energy per OFDM 


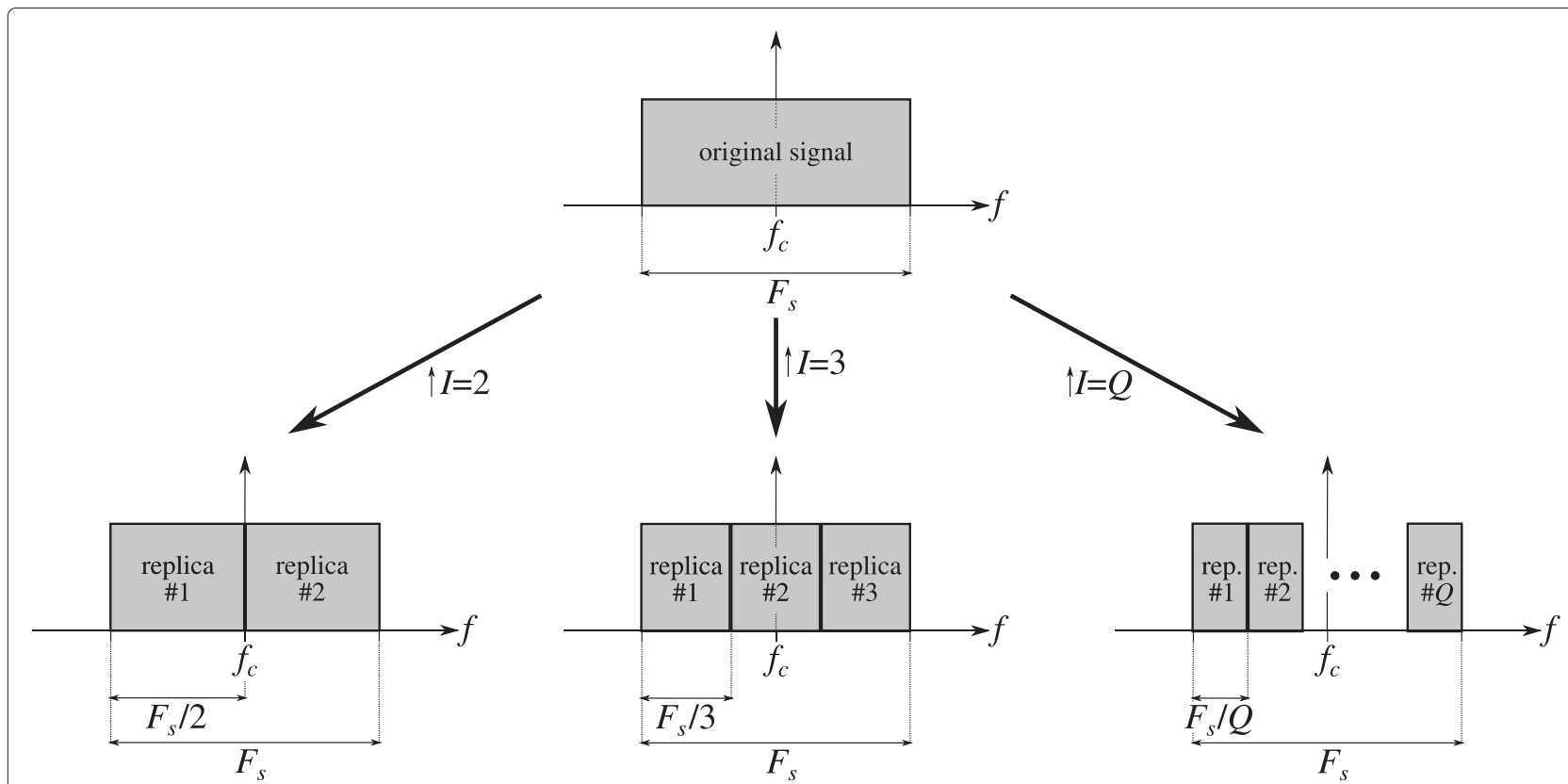

Figure 5 Ensuring equal spectrum usage for interpolation factors $I=2, I=3$, and for an arbitrary integer interpolation factor $/=Q$. $/$ replicas of the interpolated signal are transmitted to ensure that the whole frequency range of the original signal (without interpolation) is used.

symbol. Furthermore, to fairly compare the results, the channel model was fed with identical initial conditions (e.g., delays and mean power per path) for each evaluated interpolation factor. This way, we model a situation in which the receiver moves along the same path for each interpolation factor.

Table 2 details the most relevant parameters for the experimental evaluations as well as for the simulations.

\subsection{Figures of merit}

We have selected three different figures of merit that evaluate the quality of the signal at different points in the receive signal processing chain: 1) EVM calculated without knowing the transmitted symbols; and 2) uncoded BER, which is the BER after the symbol decision, and coded BER, which corresponds to the BER at the output of the channel decoder.

Recall that our main objective is to inspect the level of agreement between the results obtained from those figures of merit when the wireless system is evaluated at actual speed conditions with respect to those obtained when the system is evaluated (under similar conditions) using emulated speeds by time interpolation. Given that the testbed employed for the measurements guarantees excellent time and frequency synchronization between transmitter and receiver, the results of the three figures of merit enumerated above greatly depend on the channel equalizer, which is strongly affected by ICI.

The main reason for considering both, EVM and uncoded BER, is that EVM is an unbounded and continuous metric, which is specially valuable when the SNR levels observed are high enough to saturate the BER to its minimum value of zero. On the other hand, considering uncoded BER in the evaluation is very convenient not only because it is one of the most used performance metrics in wireless communications but also because the EVM is calculated only from the received symbols. Hence, EVM results loose accuracy as the corresponding SNR value decreases. Finally, coded BER is specially relevant in our evaluation since the proposed technique causes a signal bandwidth reduction proportional to the time interpolation factor, hence reducing the frequency diversity and potentially degrading the channel decoder performance. Consequently, one could expect that, in terms of coded $\mathrm{BER}$, the wireless system would perform differently when actual speeds are considered in comparison to emulated ones.

With the aim of evaluating of the level of agreement between the results obtained by means of actual speed with respect to emulated ones, we have included curves for the relative error values computed for those emulated speed values that can be obtained from more than one actual velocity (see Section 4). We considered the emulated speed values that can be obtained from at least two of the three interpolation factors considered (see Table 1).

The procedure followed to calculate each figure of merit is detailed below:

SNR. The SNR is estimated considering exclusively the data subcarriers. Thus, the guard subcarriers, Direct 
Current (DC) subcarrier as well as the pilots are discarded. SNR is estimated by performing the following steps:

1. Noise samples for a given velocity $v$ and interpolation factor $I$ are captured directly (measurement case) or generated (simulation case). In the measurement case, noise samples in the time domain are captured with the transmitter switched off (hence, not transmitting any signal).

2. The captured noise samples in the time domain are then processed as if they were actual data samples, i.e., they are downsampled by the corresponding interpolation factor (if required), the cyclic prefix is removed, a Fast Fourier Transform (FFT) is performed, and both guard subcarriers, pilots, and also the DC subcarrier are discarded.

3. As a result, $w^{(l, k, s)}$ noise samples are obtained in the frequency domain, each one corresponding to the l-th subcarrier of the $k$-th OFDM symbol of the $s$-th subframe and for the values of $l$ corresponding to the indexes of the data subcarriers.

4. All previous steps are repeated when the transmitter is switched on, so $r^{(l, k, s)}$ data samples are obtained in the frequency domain.

5. The average SNR for a given velocity $v$ and interpolation factor $I$ is then estimated by averaging out the SNR values corresponding to each channel realization. Defining:

$$
\bar{r}=\frac{1}{L K S} \sum_{l=1}^{L} \sum_{k=1}^{K} \sum_{s=1}^{S}\left|r^{(l, k, s)}\right|^{2},
$$

and:

$$
\bar{w}=\frac{1}{L K S} \sum_{l=1}^{L} \sum_{k=1}^{K} \sum_{s=1}^{S}\left|w^{(l, k, s)}\right|^{2},
$$

then, the SNR is calculated as follows:

$$
\mathrm{SNR}=\frac{\bar{r}-\bar{w}}{\bar{w}},
$$

with $L, K, S$ the total number of data subcarriers, OFDM symbols, and subframes, respectively.

$E V M$. The EVM is obtained based on the equalized symbols which feed the receiver decision. The following steps are considered for the EVM estimation:

1. The dynamic range of the equalized symbols is bounded. This is realistic in a practical receiver. In this sense, real and imaginary parts of the symbols are clipped to a maximum value. This avoids symbols having extremely large modulus (e.g., due to imperfect zero-forcing channel equalization), hence distorting the EVM estimation. Clipping values were selected, taking into account the mean power of the equalized symbols in perfect conditions (flat channel in the absence of noise).

2. A hard decision unit is fed with the clipped symbols. Let $\mathbf{s}_{c}=\left(s_{c}^{1}, s_{c}^{2}, \ldots, s_{c}^{S}\right)^{T}$ be the vector of $S$ clipped symbols in a subframe and $\mathbf{s}_{d}=\left(s_{d}^{1}, s_{d}^{2}, \ldots, s_{d}^{S}\right)^{T}$ the vector of decided symbols.

3. The EVM per subframe, expressed in decibels, is obtained as:

$$
\mathrm{EVM}=10 \log _{10}\left(\frac{P_{r}}{P_{c}}\right),
$$

with $P_{c}=\frac{1}{S} \mathbf{s}_{c}^{T} \cdot \mathbf{s}_{c}, P_{r}=\frac{1}{S} \mathbf{s}_{r}^{T} \cdot \mathbf{s}_{r}$, and $\mathbf{s}_{r}=\left|\mathbf{s}_{c}-\mathbf{s}_{d}\right|$. Notice that the aforementioned procedure for calculating the EVM does not require knowing the transmitted symbols. However, the higher the uncoded BER, the more underestimated the EVM is.

\section{Results}

This section presents both, measurement and simulation results. Three types of performance curves are included in the result graphs, which are:

- Red solid lines: they correspond to the cases with no interpolation $(I=1)$. According to the speed values considered for the measurements as well as for the simulations, the red solid curves range from 50 to 200 $\mathrm{km} / \mathrm{h}$.

- Green dashed lines: they correspond to the cases with $I=2$, so the emulated velocity is twice the measured or simulated speed. Therefore, the green curves range from 100 to $400 \mathrm{~km} / \mathrm{h}$.

- Pink dotted lines: they correspond to the cases where $I=3$. In this case, the emulated velocity is three times the measured or simulated speed, and the curves range from 150 to $600 \mathrm{~km} / \mathrm{h}$.

Besides the performance curves, we have also included the relative error curves for all figures although in some cases not all error curves are plotted. Three types of relative error curves are included in the graphs presenting the results (see Table 1), which are:

- Green dashed lines: they correspond to the relative difference between the results obtained when the interpolation factor $I=2$ is employed and when actual speeds are used.

- Blue dashed lines: they correspond to the relative difference of the results between the interpolation factors $I=3$ and $I=2$.

- Pink dotted lines: they correspond to the relative difference of the results between the interpolation factors $I=3$ and $I=1$ (actual speeds).

The relative error values are calculated differently for EVM and for BER (coded and uncoded): 
- Relative error for EVM: given $\mathrm{EVM}_{A}$ and $\mathrm{EVM}_{B}$ the instantaneous EVM values, corresponding to a channel realization and obtained for the interpolation factors $I=A$ and $I=B$, respectively, we define the instantaneous relative error as:

$$
E_{\mathrm{EVM}}(A, B)=100 \cdot \frac{\mathrm{EVM}_{A}-\mathrm{EVM}_{B}}{\mathrm{EVM}_{B}} \quad[\%] .
$$

- Relative error for uncoded and coded BER: given $W_{A}$ and $W_{B}$ the number of received bits estimated without errors, corresponding to a channel realization and obtained for the interpolation factors $I=A$ and $I=B$, respectively, we define the instantaneous relative error for the BER as:

$$
E_{\mathrm{BER}}(A, B)=100 \cdot \frac{W_{A}-W_{B}}{W_{B}} \quad[\%] .
$$

Mean relative error values for both error types are calculated by averaging instantaneous relative error values for all channel realizations. These mean relative error values are plotted in Figures 6, 7, 8, 9, 10, and 11 together with their corresponding $95 \% \mathrm{BCa}$ bootstrap confidence intervals for the mean [17]. These confidence intervals are plotted as an area around each curve and in the same color as the corresponding curve. We also gauge the precision of the results by calculating the $95 \%$ confidence intervals for the mean.
Due to limitations of the inverter driving the motor used to rotate the receive antenna (see Figure 3), the actual velocity range considered for the measurements (and also for the simulations) starts at $50 \mathrm{~km} / \mathrm{h}$, while the maximum actual speed value is set to $200 \mathrm{~km} / \mathrm{h}$. Given that we consider interpolation factors $I=1,2,3$, in the speed range from 50 to $300 \mathrm{~km} / \mathrm{h}$, at least two curves overlap, thus allowing for evaluating the level of agreement of the results between different interpolation factors.

\subsection{Measurement results}

Figure 6 shows the measured EVM for CQI $=12$ (64QAM) for SNR values ranging from 38 to $11 \mathrm{~dB}$. For high SNR values, it can be seen that the higher the emulated speed is, the worse the EVM becomes, as expected. However, it can be seen that the curves for different interpolation factors (as well as their associated confidence regions) overlap, which demonstrates that the proposed technique of emulating high speeds by time interpolation performs adequately. Results for CQI $=1$ (4-QAM) and CQI $=8$ (16-QAM) were not included, but they have been also evaluated, and they show that the achieved performance is very similar despite the considered modulation scheme. Figure 6 also shows that the quality of the received signal, in terms of EVM, is considerably affected by the speed for high SNR values, showing that the main source contributing to the signal distortion is the ICI. As the SNR decreases, however, the performance is less affected by the

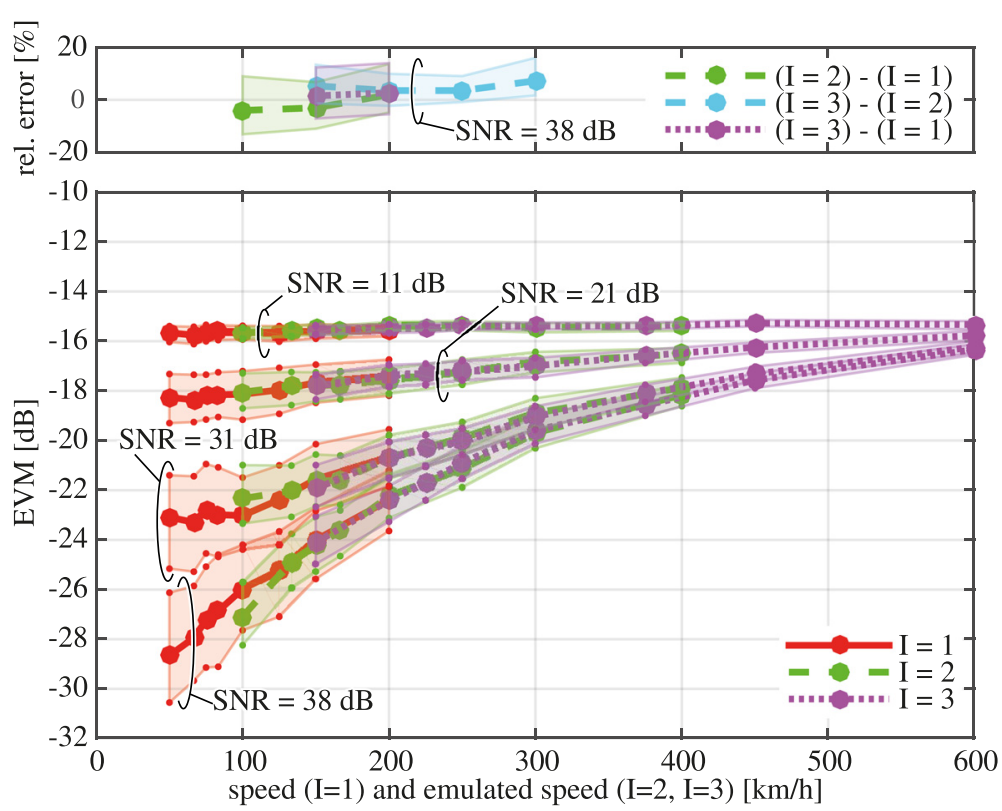

Figure 6 Measured EVM for different SNR values. EVM results obtained by measuring when CQI = 12 and SNR ranges from 11 to $38 \mathrm{~dB}$ for the interpolation factors $I=1,2,3.95 \%$ confidence regions are provided. We have also evaluated the EVM for CQI $=1$ (4-QAM) and CQI = $8(16-\mathrm{QAM})$, and the results are almost indistinguishable. Corresponding relative error curves are plotted when the SNR is set to $38 \mathrm{~dB}$, showing an excellent agreement. 

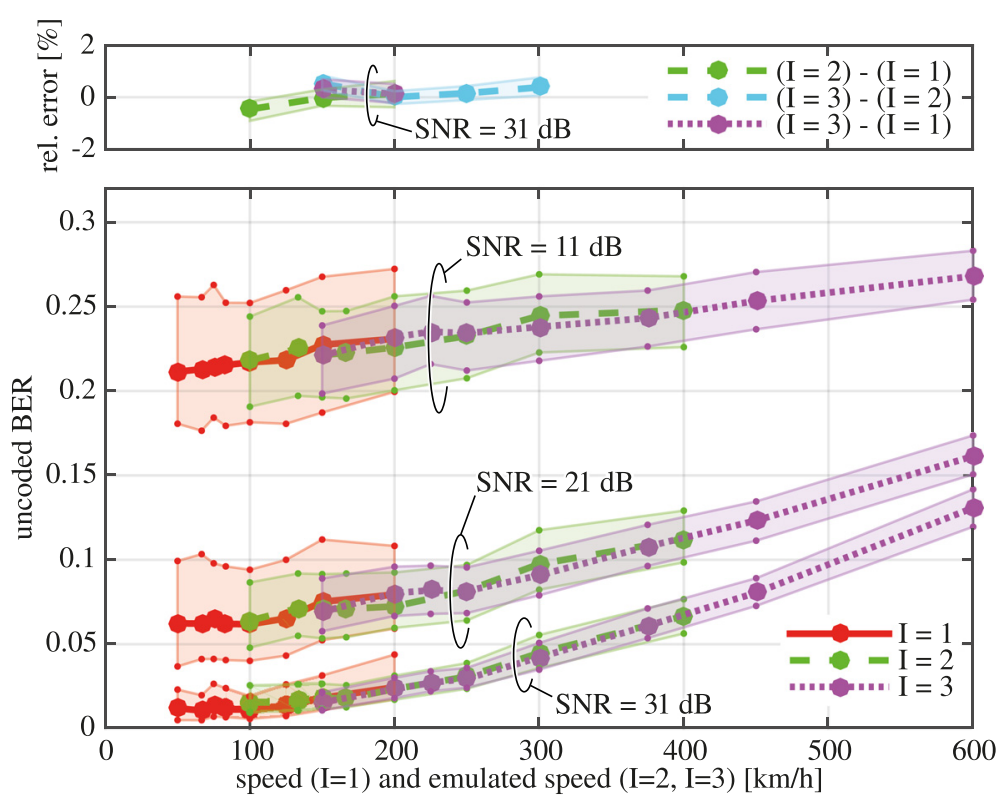

Figure 7 Measured uncoded BER for different SNR values. Uncoded BER results obtained by measuring when CQl = 12 and SNR ranges from 11 to $31 \mathrm{~dB}$ for the interpolation factors $/=1,2,3.95 \%$ confidence regions are provided. Corresponding relative error curves are plotted when the SNR is set to $31 \mathrm{~dB}$, showing an excellent agreement.

emulated speed because the noise is the main contributor to the signal distortion.

The relative error curves in Figure 6 also demonstrate the excellent agreement of the results independently of the source of the Doppler spread: actual speed or emulated velocity from time interpolation. Note that the confidence intervals for the relative error values are mainly influenced by the number of realizations averaged. Hence, these confidence intervals could be decreased by increasing the number of realizations measured. However, as the

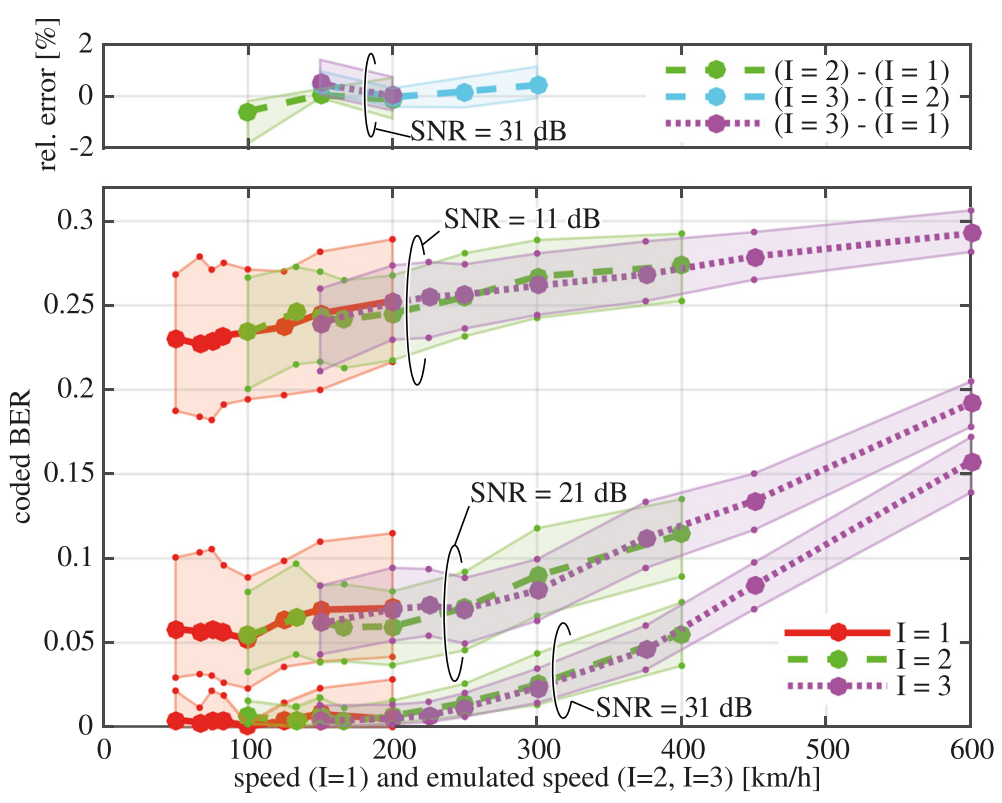

Figure 8 Measured coded BER for different SNR values. Coded BER results obtained by measuring when CQI = 12 and SNR ranges from 11 to $31 \mathrm{~dB}$ for the interpolation factors $I=1,2,3.95 \%$ confidence regions are provided. Corresponding relative error curves are plotted when the SNR is set to $31 \mathrm{~dB}$, showing an excellent agreement. 


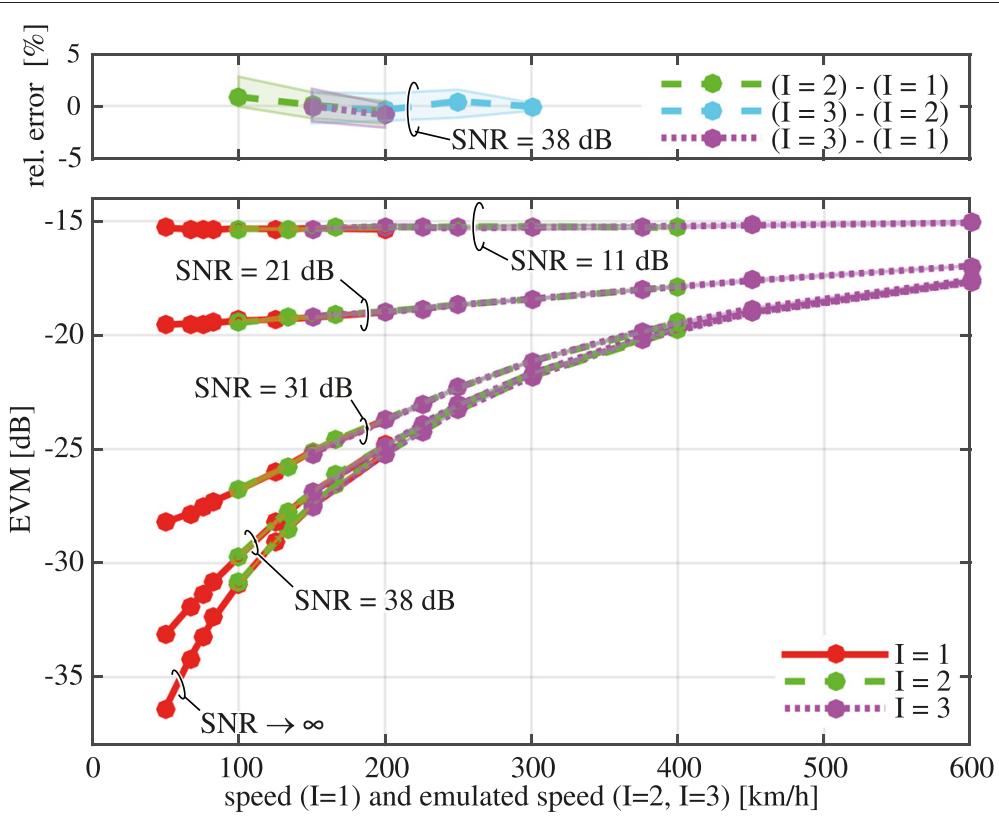

Figure 9 Simulated EVM for different SNR values. EVM results obtained by simulation when CQI $=12$ and SNR ranges from $11 \mathrm{~dB}$ to infinity for the interpolation factors $I=1,2,3$. Note that the channel model associated to the D2a link of the D2 scenario of the Winner Phase II Channel Models was applied. 95\% confidence regions are provided. We have also evaluated the EVM for CQI = 1 (4-QAM) and CQI = 8 (16-QAM), and the results are almost indistinguishable. Corresponding relative error curves are plotted when the SNR is set to $38 \mathrm{~dB}$, showing an excellent agreement.

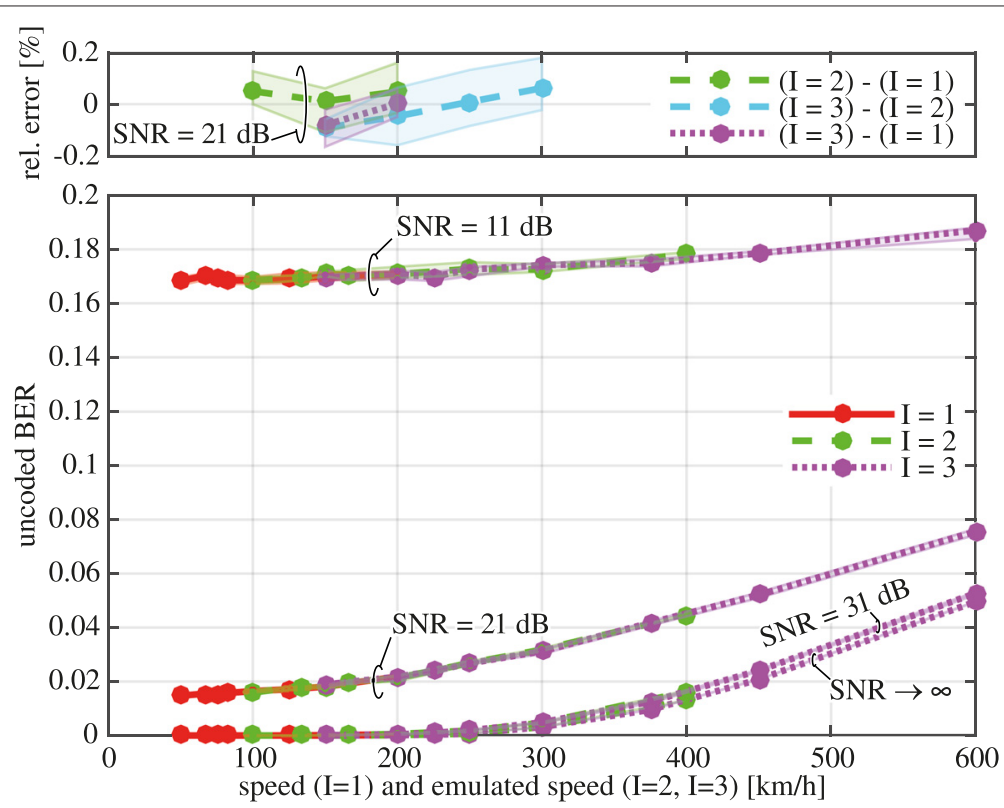

Figure 10 Simulated uncoded BER for different SNR values. Uncoded BER results obtained by simulation when CQI $=12$ and SNR ranges from 11 to $31 \mathrm{~dB}$ for the interpolation factors $I=1,2,3$. Note that the channel model associated to the D2a link of the D2 scenario of the Winner Phase II Channel Models was applied. 95\% confidence regions are provided. Corresponding relative error curves are plotted when the SNR is set to $21 \mathrm{~dB}$, showing an excellent agreement. Note that for SNR values greater or equal than $31 \mathrm{~dB}$, the BER curves are close to zero for speeds below $250 \mathrm{~km} / \mathrm{h}$; hence, the relative error curves do not provide much information. 


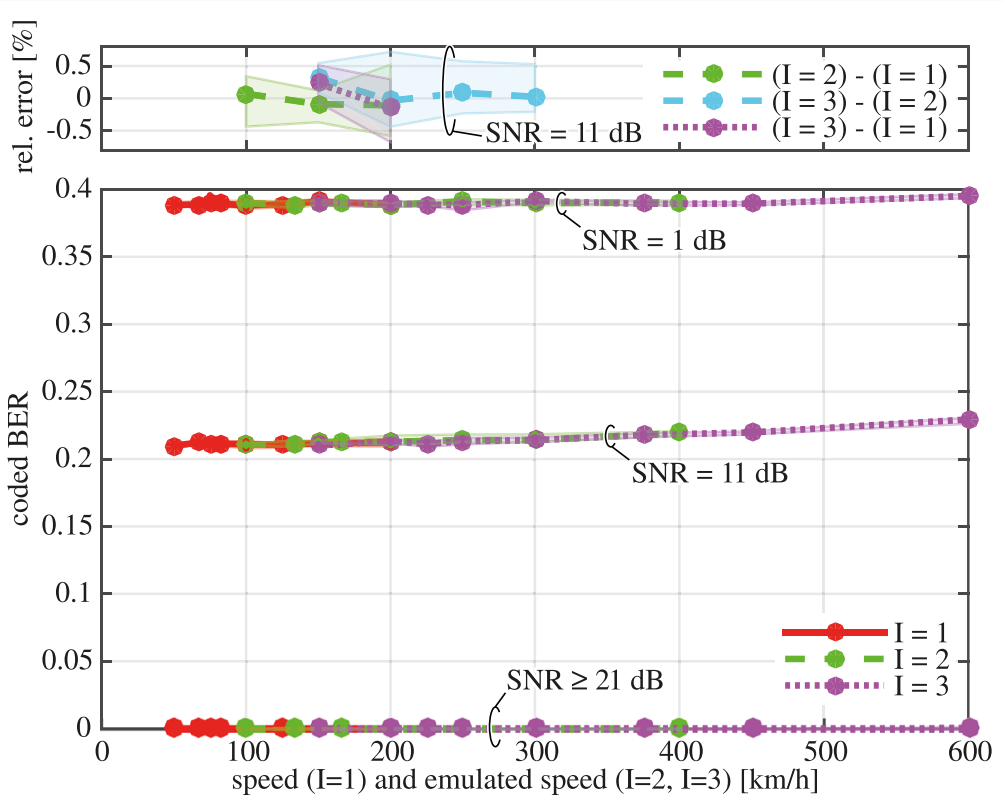

Figure 11 Simulated coded BER for different SNR values. Uncoded BER results obtained by simulation when CQI = 12 and SNR ranges from $1 \mathrm{~dB}$ to infinity for the interpolation factors $I=1,2,3$. Note that the channel model associated to the D2a link of the D2 scenario of the Winner Phase II Channel Models was applied. $95 \%$ confidence regions are provided. Corresponding relative error curves are plotted when the SNR is set to $11 \mathrm{~dB}$, showing an excellent agreement. Note that for SNR values greater or equal than $21 \mathrm{~dB}$, the BER curves are close to zero for all considered speeds; hence, the relative error curves do not provide much information.

receive antenna trajectory is kept constant between different interpolation factors, the relative error is mainly influenced by the repeatability of the setup, the noise, and the interpolation itself. For this reason, we can achieve relative agreements with mean error values below $10 \%$ in the worst case (i.e., the highest SNR value of $38 \mathrm{~dB}$ ) without increasing the number of channel realizations.

Figures 7 and 8 show the measured uncoded BER and coded BER, respectively, for different SNR values when $\mathrm{CQI}=12$. Both, uncoded BER and coded BER, are strongly dependent on the emulated speed for high SNR values, while the noise is the main source of signal distortion when the SNR decreases. It can also be observed that curves for different interpolation factors overlap both for uncoded BER and coded BER, demonstrating again the excellent results of the proposed technique. Notice also that, as shown in the corresponding relative error curves in Figures 7 and 8, the agreement of the proposed technique is below $2 \%$, both in terms of uncoded BER and coded BER. Therefore, such a proposed technique can be effectively used for emulating high-speed effects at any point of the receive signal processing chain.

\subsection{Simulation results}

The channel model of the simulations was selected because of its suitability for the simulation of high-speed conditions. Notice that such a channel model leads to frequent LoS propagation conditions [3]. However, the receiver in the measurements is located inside a building, while the eNodeB is placed on a roof of another building (see Figure 2), and hence, NLoS propagation conditions arise, leading to a much more relevant multipath effect.

Figure 9 shows the simulated EVM for CQI $=12$ (64QAM) for SNR values ranging from 38 to $11 \mathrm{~dB}$. Additionally, we have also evaluated the results in the absence of noise (infinite SNR). Note that the slopes of the curves are similar to those obtained by measurements as shown in Figure 6, while the obtained EVM values are always lower for the same SNR value. This result is expected because the channel model considered for the simulations is easier to equalize due to the lower variability of the simulated channel. However, when the SNR decreases ( $\mathrm{SNR}=11 \mathrm{~dB}$ and SNR $=21 \mathrm{~dB}$ in our case), the abovementioned effect vanishes as the noise becomes the main contribution to the signal distortion and the corresponding curves approach each other for simulations and for measurements.

The variability of the results obtained by simulations is much lower than that of the measurements because, on the one hand, the simulated channel is less variable than that observed in the measurements. On the other hand, exactly the same initial channel conditions are applied in the simulations for each interpolation factor and velocity pair, thus ensuring much less variability between the different channel realizations generated for each interpolation factor and velocity pair. In fact, the confidence region 
of each simulated curve completely overlaps the curve itself, which causes that the confidence regions cannot be appreciated although they are included. Additionally, curves simulated for different interpolation factors almost completely overlap, hence showing the good behavior of the proposed technique also in this case. This precise behavior is also confirmed by the corresponding relative error curves in Figure 9.

Finally, Figures 10 and 11 show the simulated uncoded BER and coded BER, respectively, for different SNR values when $C Q I=12$. Some level of dependency on the emulated speed for high SNR values can be still appreciated for the uncoded BER, while the coded BER is always zero for SNR values greater than or equal to $21 \mathrm{~dB}$ since the channel decoder is able to correct all errors. Notice that this is not the case for the measurements as the channel is much more difficult to equalize. Curves for different interpolation factors overlap both for uncoded BER and coded BER, thus showing the good behavior of the proposed interpolation technique. This is also confirmed by the corresponding error curves.

\section{Conclusions}

We have shown that time interpolating OFDM signals prior to their transmission followed by the corresponding decimation at the receiver is a suitable technique for inducing high-speed effects (mainly ICI) while actually measuring at much lower speeds. The key idea behind the proposed technique is that the ICI level experienced by the received signals after OFDM demodulation and before channel equalizer depends on the relative factor between subcarrier spacing and Doppler spread; thus, instead of changing the Doppler spread, one can change the subcarrier spacing by the same factor to obtain the same results. The main advantage of the proposed technique is its feature to offer experimental evaluation of OFDM-based wireless communication systems at very high velocities while conducting measurements at much lower speeds. The price to be paid is that the signal bandwidth is reduced proportionally to the time interpolation factor, and therefore, potential inaccuracies could arise due to the loss of frequency diversity. However, to combat this loss in diversity, we proposed to transmit different replicas of the interpolated signal to cover the same bandwidth as in the non-interpolated case.

We have designed a measurement methodology for validating our technique in a controlled environment and under repeatable conditions. The basic idea consists in evaluating different figures of merit for a certain range of actual speed values and to compare them with emulated speeds of the same magnitude under the same conditions. For example, when the figures of merit considered are evaluated at $150 \mathrm{~km} / \mathrm{h}$, three different cases are evaluated under the same conditions: 1 ) the receive antenna moves at the actual speed of $150 \mathrm{~km} / \mathrm{h} ; 2)$ the receive antenna moves at the actual speed of $75 \mathrm{~km} / \mathrm{h}$ and a time interpolation factor $I=2$ is used, hence the emulated speed is $75 \times 2=150 \mathrm{~km} / \mathrm{h}$; and 3) the receive antenna moves at $50 \mathrm{~km} / \mathrm{h}$, and $I=3$ is applied. Besides the measurement results, we have also evaluated the proposed technique through simulations based on a channel model specifically designed for modeling high-speed scenarios. We selected EVM, uncoded BER, and coded BER as figures of merit, all being evaluated for different SNR, CQI, and speed values. All results based on the three figures of merit have shown an excellent agreement between actual and emulated speeds for all the interpolation factors considered, hence validating the proposed technique as a good candidate to be considered not only for physical layer performance evaluations but also for higher layer ones.

Besides validating the proposed technique, we have also shed light on the effects caused by high and even extremely high speeds on OFDM signals. More specifically, we have selected LTE as a waveform example of the state of the art of current mobile wireless systems. As expected beforehand, received signals suffer a similar degradation (in terms of EVM, uncoded BER, and coded BER) when the SNR decreases or when the speed (and correspondingly the ICI level) increases. However, low SNR values can conceal the effects due to high speeds as the noise becomes the main contributor of the signal distortion. We have shown that the level of signal distortion (measured in terms of EVM) caused by high-speed conditions does not depend on the modulation scheme.

We have also shown that the proposed technique performs excellent not only in outdoor-to-indoor measurement scenarios but also in simulations for a channel model specifically designed for outdoor-to-outdoor high-speed scenarios. In both cases, the level of agreement between the results for actual speed and for emulated speeds is at the level of possible measurement accuracy, as the corresponding relative error curves have shown.

Finally, in the light of the excellent agreement of the results obtained under repeatable conditions for the three figures of merit considered (EVM, uncoded BER, and coded BER) and regardless actual or emulated speeds are considered, it can be concluded that the proposed technique is valid for inducing high-speed effects at any point in the signal processing chain at the receiver.

\section{Competing interests}

The authors declare that they have no competing interests.

\section{Acknowledgements}

This work has been funded by the Christian Doppler Laboratory for Wireless Technologies for Sustainable Mobility, KATHREIN Werke KG, and A1 Telekom Austria AG. This work was supported in part by the Xunta de Galicia, MINECO of Spain, and FEDER funds of the E.U. under Grant 2012/287, Grant IPT-2011-1034-370000, Grant TEC2013-47141-C4-1-R, Grant FPU12/04139, and Grant EST13/00272. The financial support by the Austrian Federal Ministry of 
Economy, Family and Youth and the National Foundation for Research, Technology and Development is gratefully acknowledged.

\section{Author details}

${ }^{1}$ Department of Electronics and Systems, University of A Coruña, Facultade de Informática, Campus de Elviña, A Coruña 15071, Spain. ${ }^{2}$ Institute of Telecommunications, TU Wien, Gusshausstrasse 25/389, A-1040 Vienna, Austria.

Received: 13 December 2014 Accepted: 25 March 2015

Published online: 18 April 2015

\section{References}

1. Radiocommunication Sector of International Telecommunication Union (ITU-R), Guidelines for evaluation of radio transmission technologies for IMT-2000. ITU-R Recommendation M.1225 (1997)

2. 3GPP TSG-RAN Working Group 4 (Radio), High speed environment channel models (R4-050388). 3GPP TSG-RAN Working Group 4 (Radio) meeting \#35, (Athens, Greece, 2005)

3. P Kyosti, J Meinila, L Hentila, X Zhao, T Jamsa, C Schneider, M Narandzic, M Milojevic, AHJ Ylitalo, Veli-Matti, Holappa, M Alatossava, R Bultitude, Y de Jong, T Rautiainen, IST-4-027756 WINNER II D1.1.2 V1.1: WINNER II Channel Models (2007)

4. Radiocommunication Sector of International Telecommunication Union (ITU-R), Guidelines for evaluation of radio interface technologies for IMT-Advanced, Report ITU-R M.2135-1 (2009)

5. H Wei, Z Zhong, K Guan, B Ai, in 2010 IEEE 5th International ICST Conference on Communications and Networking in China. Path loss models in viaduct and plain scenarios of the high-speed railway (IEEE, USA Beijing, China, 2011), pp. 1-5

6. K Guan, Z Zhong, B Ai, in 2011 IEEE Third International Conference on Communications and Mobile Computing (CMC). Assessment of LTE-R using high speed railway channel model (IEEE,USA, Quingdao, China, 2011), pp. 461-464. doi:10.1109/CMC.2011.34

7. L Liu, C Tao, T Zhou, Y Zhao, X Yin, H Chen, A highly efficient channel sounding method based on cellular communications for high-speed railway scenarios. EURASIP J. Wireless Commun. Netw. 2012(1), 1-16 (2012)

8. J Rodríguez-Piñeiro, JA García-Naya, A Carro-Lagoa, L Castedo, in 2013 Euromicro Conference on Digital System Design. A testbed for evaluating LTE in high-speed trains (IEEE, USA, Santander, Spain, 2013), pp. 175-182. doi:10.1109/DSD.2013.27

9. Rodríguez-Piñeiro, J, P Suárez-Casal, JA García-Naya, L Castedo, C Briso-Rodríguez, II Alonso-Montes, in 2014 IEEE Eighth IEEE Sensor Array and Multichannel Signal Processing Workshop. Experimental validation of ICl-aware OFDM receivers under time-varying conditions (IEEE, USA, A Coruña, Spain, 2014). doi:10.1109/SAM.2014.6882411

10. Z Wang, GB Giannakis, Wireless multicarrier communications. IEEE Signal Process. Mag. 17(3), 29-48 (2000). doi:10.1109/79.841722

11. C Mehlführer, JC Ikuno, M Simko, S Schwarz, M Wrulich, M Rupp, The Vienna LTE simulators - enabling reproducibility in wireless communications research. EURASIP J. Adv. Signal Process. 2011, 1-13 (2011)

12. M Lerch, S Caban, M Mayer, M Rupp, The Vienna MIMO testbed: evaluation of future mobile communication techniques. Intel Technol. J. 18(3), 58-69 (2014)

13. S Caban, JA Garcia-Naya, M Rupp, Measuring the physical layer performance of wireless communication systems: part 33 in a series of tutorials on instrumentation and measurement. IEEE Instrum. Meas. Mag. 14(5), 8-17 (2011). doi:10.1109/MIM.2011.6041377

14. S Caban, A Disslbacher-Fink, JA Garcia-Naya, M Rupp, in Proc. International Instrumentation and Measurement Technology Conference (I2MTC 2011). Synchronization of wireless radio testbed measurements (IEEE, USA Binjiang, Hangzhou, China, 2011). doi:10.1109/IMTC.2011.5944089

15. S Caban, R Nissel, M Lerch, M Rupp, in Proc. of 6th Extreme Conference on Communication and Computing (ExtremeCom). Controlled OFDM measurements at extreme velocities (Association for Computing Machinery (ACM), USA San Cristobal, Galapagos, Ecuador, 2014)
16. S Caban, J Rodas, JA Garcia-Naya, in 2011 IEEE Instrumentation and Measurement Technology Conference. A methodology for repeatable, off-line, closed-loop wireless communication system measurements at very high velocities of up to $560 \mathrm{~km} / \mathrm{h}$ (IEEE, USA Binjiang, China, 2011), pp. 1-5. doi:10.1109/IMTC.2011.5944019

17. B Efron, DV Hinkley, An Introduction to the Bootstrap (CRC Monographs on Statistics \& Applied Probability), 1st edn. (Chapman \& Hall, USA, 1994)

\section{Submit your manuscript to a SpringerOpen ${ }^{\mathcal{O}}$ journal and benefit from:}

- Convenient online submission

- Rigorous peer review

- Immediate publication on acceptance

- Open access: articles freely available online

- High visibility within the field

- Retaining the copyright to your article

Submit your next manuscript at $\gg$ springeropen.com 